\title{
Astro Concepts: Learning Underlying Physics Principles in Conceptual Astronomy
}

\author{
Margaret Mazzolini and Bronwyn Halls \\ Centre for Astrophysics and Supercomputing, BSEE, Mail Box 31, \\ Swinburne University of Technology, PO Box 218, Hawthorn, Vic. 3122, Australia \\ mmazzolini@swin.edu.au \\ Received 1999 August 17, accepted 2000 May 2
}

\begin{abstract}
Astro Concepts is a project within Swinburne University of Technology in Melbourne developing browser-based software modules on Optical Telescopes, Nebulae and Binary Stars. The modules are designed to enhance students' understanding of basic physics concepts, which underlie introductory-level conceptual astronomy courses. When complete, the Astro Concepts modules will be available for use in university courses in introductory astronomy, introductory physics teaching, secondary teaching and online astronomy education. The strategy outlined here is to obtain a reasonable level of understanding of the necessary physics concepts by presenting them embedded in relevant and interesting astronomy contexts, and by the use of an engaging educational approach requiring active learning by the student.
\end{abstract}

Keywords: astronomy education

\section{Introduction and Rationale}

In this paper we describe the Astro Concepts Project* that is presently under development. The aim of the project is to develop freeware software-teaching modules on three topics: Optical Telescopes, Nebulae, and Binary Stars.

Introductory-level, conceptual astronomy units, usually offered with little or no mathematics or physics prerequisites, are extremely popular at Australian universities. Conceptual astronomy can be taught successfully with little mathematics, but some understanding of basic physics is necessary even at this level.

A conventional approach is to teach basic physics concepts as separate isolated topics (usually) at or near the start of an astronomy lecture course. This is an ineffective way of remedial teaching in physics: even mainstream physics students learn relatively little in lectures (Hestenes, Wells \& Swackhamer 1992; Redish 1995). An alternative approach is to use laboratory classes to teach remedial physics concepts; however, either approach is likely to be unpopular. Students object that they have enrolled to learn astronomy, not physics. This negative attitude inhibits their learning, and they have difficulty connecting briefly covered abstract physics concepts to the rest of their astronomy course.

A teaching approach where physics principles are thoroughly integrated with astronomy contexts is likely to be much more readily accepted by students. This approach can be used in lectures, but is more likely to be effective in a less formal setting (such as a laboratory class) and when combined with an engaging educational approach which encourages active learning.

Educational research in physics has shown that fundamental misconceptions about physics principles are very robust (Van Heuvelen 1991; McDermott 1991). Missing years of physics training cannot be replaced with a few hours of remedial teaching, and computer animations are no substitute for extended laboratory work; however, the latter is rarely a practical option in astronomy courses. On the other hand, conceptual astronomy courses usually aim to teach a general audience about astronomy rather than to produce astronomers (or physicists). In this case, a little basic but well understood physics can go quite a long way! For this reason we believe that educational software which aims to use an engaging approach and active learning techniques whilst presenting physics concepts embedded in interesting astronomy contexts might prove helpful.

In this project we are developing web browser-based Astro Concepts software modules designed to promote active learning of underlying physics concepts in astronomy contexts. Each engages the students in actively studying an astronomy context chosen to elucidate underlying physics concepts that are fundamental to understanding astronomy. In each case, the context focuses on a particular astronomy topic but, in the process, the concepts being taught are basic physics principles. In each module students work through background material, simulations and videos (where appropriate). In the process they are challenged to make predictions, check them and then use the results to refine their understanding. The modules could be assigned as computer-based laboratory exercises or as individual self-study exercises, and are based on experience gained in teaching astronomy at three universities and in surveying students to probe their understanding of basic astronomy concepts.

The modules draw on simulations, videos, assessment exercises and optional practical exercises where

\footnotetext{
* Margaret Mazzolini coordinates the Astro Concepts Project and Bronwyn Halls is its Curriculum Development Officer.
} 
appropriate, and are designed to be deliverable via the Internet, local area networks, or CDROM. After trialing with student groups studying astronomy at several universities, the modules and support material will be made available to Australian universities. The modules will also be suitable for use in online education.

\section{The Modules}

Each module is designed within an astronomy context that has been chosen in order to focus on a particular set of basic but important underlying physics concepts. The three areas of physics concepts that have been chosen are geometric optics, light and atomic spectra, and gravity. The astronomy contexts chosen are, respectively, Optical Telescopes, Nebulae, and Binary Stars. These topics occur in most astronomy courses and are natural contexts in which to study the chosen physics concepts.

The original brief was to produce enough material for three modules in the time available-one on each of the contexts listed above. However; the amount of useful teaching material that has been produced so far is such that we have tentatively divided it up into two modules per context. The modules have not been tested with students yet. It is planned that this will happen in the second half of 2000. The final split into modules will be decided in the light of the amount of time most students take to work through the material, but we are confident that we will end up with six useful modules once complete assessment details have been included. Some instructors may prefer that we keep the number of modules to a minimum. However, we believe that splitting the material up into pairs of modules with, in each case, a relatively general introductory module followed by more advanced one will provide them with greater choice where classes with different levels of background knowledge of physics are involved.

\subsection{The Binary Stars Modules}

The study of binary systems is used in these modules as a vehicle for the introduction of laws governing classical descriptions of motion up to and including Kepler's Third Law. The first binary stars module (working title, Binary Stars A) uses simulations of binary star systems with differing stellar masses and orbit shapes to introduce and illustrate qualitatively Newton's Law of Gravitation and Kepler's 1st and 2nd laws. The second module (Binary Stars B) seeks a qualitative explanation for visual binary motions and light curves and spectra for eclipsing and spectroscopic binaries, in the simplest cases of circular orbits viewed edge-on. For visual binaries, a simulation relates motion on the sky to motion relative to the centre of mass. Examples of differing separations and periods illustrate Kepler's 3rd law. For eclipsing binaries, students are guided to form a qualitative model relating the light curve to eclipses and then test it with an interactive simulation using various relative stellar brightnesses and sizes. For spectroscopic binaries, a Doppler shift simulation aids students to form and test a qualitative model relating spectral Doppler shifts with approaching and receding motion of the stars in their orbits.

\subsection{The Nebulae Modules}

These modules use optical images of spectacular emission, reflection and dark nebulae plus examples of interstellar absorption, as an astronomy context in which to introduce students to the study of the reflection, transmission and emission of light, including the fundamentals of spectroscopy. In the first module (Nebulae A), which concentrates on the transmission and reflection of light, students investigate how nebulae appear to astronomers, how light reaches Earth from and through nebulae, and study a model of light that explains the colour effects. The second module (Nebulae B) concentrates on the emission of light, discussing what a spectrum is and the three types of spectra (continuous, emission and absorption), how spectra are formed and observed, the relevance of spectra to astronomy and the basics of spectroscopy.

\subsection{The Optical Telescopes Modules}

In these modules, students are guided to a qualitative understanding of the basic design of optical telescopes, as they help Lee, a very innocent would-be amateur astronomer, decide what sort of telescope to buy. They investigate the images formed by both mirrors and lenses via an instructional technique that avoids the need to teach the construction of ray diagrams at this stage. They are then guided to combine their findings in order to come to a qualitative understanding of how refracting and reflecting telescopes form and magnify images.

The first module (Optical Telescopes A) concentrates on mirrors and lenses. Students learn about image formation by plane and concave mirrors, then by convex lenses. They either perform experiments (where equipment is available) or work though simulations provided in the module. In the second module (Optical Telescopes B), students investigate theoretically how mirrors and lenses can be put together to build a basic telescope that will provide bright clear images that are easy to record.

\section{Intended Applications}

We have designed the modules for the following four applications: university courses in introductory astronomy, introductory physics teaching, secondary teaching and online astronomy education.

Introductory astronomy courses often have little or no access to conventional physics laboratory equipment, but may have access to 'computer laboratories', especially given the widespread use of the CLEA Project* astronomy simulation software in these courses. The 
Astro Concepts modules will be suitable for use in 2 or 3 hour long computer laboratory sessions as part of conventional introductory astronomy courses, either for the whole student group or for those with little or no physics background.

The fact that these modules teach physics principles within astronomy contexts does not restrict their use to astronomy courses: they are also intended to provide an engaging approach suitable for use in introductory physics courses.

Suitably edited versions of these modules would be suitable for use in upper secondary education, as they concentrate on basic principles and avoid the use of specialist astronomical language and situations.

Participants in online astronomy courses have frequently studied little or no physics, or have last studied physics a number of years ago. Typically they are studying astronomy for interest rather than as a pathway to professional astronomy. For example, this is the case for most participants in the online astronomy courses run by Swinburne University in which the authors are involved. Given that the online mode of studying astronomy is largely computer-based, the software Astro Concepts modules should fit naturally into these programs.

\section{Software Format}

The modules are being developed as fully animated PowerPoint 97 presentations and will be made available as 'freeware' to astronomy educators. A short example of part of the Optical Telescopes Module is available at

http://www.swin.edu.au/astronomy/astroconcepts/

Because the modules are being written in PowerPoint 97, they will be easily downloaded and played on both Windows/NT and Macintosh platforms using the (freeware) PowerPoint 97/98 player.

As well as the basic animated software format, where relevant the modules will suggest practical activities (for teaching situations where equipment is available), provide simulations (for where equipment is not available), plus videos, assessment tasks and instructors' notes.

The PowerPoint 97 format will allow astronomy instructors to customise the modules, where appropriate, to suit their particular needs. The Astro Concepts Project will waive copyright restrictions on modifying project material, as long as the project labels are not removed. Instructors who modify modules for particular educational purposes will be asked to make copies of the modified modules available to the project, together with a description of the reasons for the modifications (e.g. to shorten module, to adapt for secondary school use, etc.). They should also list the intended use (e.g. upper secondary, introductory university physics, etc.). The intention is to build up a download area on the project website containing different versions of the modules, together with descriptions of intended use and contact details for the author of the modifications.

\section{Current Status}

At the time of writing (February 2000), authoring of the teaching content of the Optical Telescopes and Nebulae Modules is essentially complete, and the Binary Stars Modules are mostly completed. The modules already contain formative self-test multiple-choice questions and answers. What remains to be done before betatesting and proper evaluation can take place is the design and inclusion of more formal multiple-choice and extended explanation questions, which could be used by instructors to test student understanding. The instructors' notes have not yet been written and the video segments of telescopes have not yet been made. We expect to have completed these tasks by June 2000.

\section{Conclusion}

The Astro Concepts Project involves the development of up to six software modules designed to promote active learning of underlying physics concepts in astronomy contexts. The modules are being authored in PowerPoint 97, which will allow instructors to customise their content, where desired. Once beta testing and trialling is complete, the modules will be freely downloadable in early 2000 from the Astro Concepts Project website given above.

\section{Acknowledgments}

This project is supported by a CUTSD (Committee for University Teaching and Staff Development) Individual Grant awarded jointly to Swinburne University of Technology and La Trobe University for 1999 . $^{*}$

\section{References}

Hestenes, D., Wells, M., \& Swackhamer, G. 1992, The Phys. Teacher, 30, 141

McDermott, L. C. 1991, Am. J. Phys., 59, 301

Redish, E. F. 1995, Research motivated methods of instruction using computers. OzCUPE2 Conf. on Computers in Physics Education in Australia, Melbourne

Van Heuvelen, A. 1991, Am. J. Phys., 59, 891 